\title{
Survey Paper on Clustering based Segmentation Approach to Detect Brain Tumour from MRI Scan
}

\author{
Upasana Gaikwad \\ IT Department \\ Dr.D.Y.Patil Institute of \\ Engineering and Technology, \\ Ambi, Pune
}

\author{
Kanika Debbarma \\ IT Department \\ Dr.D.Y.Patil Institute of \\ Engineering and Technology, \\ Ambi, Pune
}

\author{
Silkesha Thigale \\ IT Department \\ Dr.D.Y.Patil Institute of \\ Engineering and Technology, \\ Ambi, Pune
}

\begin{abstract}
In the last few years, many image processing techniques have been presented in order to perform different brain tumor detection tasks. These cover Content-Based Retrieval Technique, Component Labelling Algorithm, Fuzzy C-Mean Algorithm. There is fast growth of image processing available in last few years; image segmentation have work together to get extraction of meaningful data into useful information. Image processing is used to finding applicable and efficient information from data available. With this survey paper we focus on literature survey of segmentation based image processing model as contain efficient performance.
\end{abstract}

\section{Keywords}

biomedical, k-means algorithm, magnetic resonance images, nervous system, spinal cord, skull.

\section{INTRODUCTION}

In the last few years, many image processing techniques have been presented in order to perform brain tumor detection and segmentation task in magnetic resonance image(MRI).These covers Knowledge-Based Technique, Component Labeling Algorithm, Content-Based Retrieval Method, Fuzzy C-means Algorithm, PNN(Probabilistic Neural Network) Technique, Computer Aided System. In biomedical science the brain tumor detection and segmentation is a very important task. there is fast growth of the image processing techniques available in last few years, biomedical terms and image processing have work together to get the actual and accurate result of the brain tumor. Magnetic resonance imaging (MRI) has become a widely-used method of high-quality medical imaging, mostly in brain imaging and MRI's soft tissue contrast and noninvasiveness are clear advantages[10]. Most of the current conventional diagnosis techniques are based on human experience in interpreting the MRI-scan for judgment; certainly this increases the possibility to false detection and identification of the brain tumor[12].With this survey paper we focus on literature survey of brain tumor detection and segmentation techniques. The benefits of brain tumor detection and segmentation technique is higher accuracy and efficiency.

\section{LITERATURE SURVEY REVIEW}

\subsection{Knowledge-based Technique}

In this paper, system that automatically segments and uses the labels glioblastoma-multiforme tumors in magnetic resonance images (MRI's) of the human brain is presented[10].Along with cluster, the segmented images find outs the centers for each class that are provided to a rule-based expert system which extracts the region of intracranial. It uses the technique of Multispectral histogram analysis separates suspected tumor from the rest of the region of intracranial, with region analysis used in performing the final tumor labeling. Computer-based brain tumor segmentation. Many efforts have exploited MRI's multidimensional data capability through multispectral analysis.

\subsection{Component Labelling Algorithm}

In this paper, An image segmentation scheme to segment 3D brain tumor from MRI images through the clustering process[11]. The clustering is achieved using K-mean algorithm in conjunction with the connected component labeling algorithm to link the similar clustered objects in all 2D slices and then obtain 3D segmented tissue using the patch object rendering process. It is allowing a diagnostics automation and assist the expert in the qualitative and quantitative analysis. It is very important for the surgical planning, and quantitative analysis such as volume measurements. In existing system it uses K-mean 2D Slice Clustering It consists in applying to each slice the k-mean clustering algorithm in $2 \mathrm{D}$ and to propagate the result by labeling/relabeling the clustered contours of the preceding slice.

\subsection{Content based Retrieval Method}

In this paper the Content-based medical image retrieval allows exploring same images appearance with the different kind of diagnosis. It also allows the searching through a large collections of disease-related illustrations using the visual attributes. It provides a convenient environment for the retrieved images[8]. Approaches that are based on one-to-one regions of image matching for shifting and scaling. The existing system uses the MR images using discrete wavelet transformation (DWT).Following that, principles component analyses (PCA) that were use to reduce the features of MR image.

\subsection{Fuzzy C-Means Algorithm}

This paper deals with the implementation of Simple Algorithm for detection of range and shape of tumor in brain MRI. Tumor is an uncontrolled growth of tissues in any part of the body[6].Tumors are of different types and they have different Characteristics and different treatment. The advantage is that in existing method is based on the thresholding. The thresholding technique was ignored the spatial characteristics. This technique consist of segmentation, pre-processing, Feature extraction, and approximate reasoning. This project uses computer aided method for segmentation and detection of brain tumor based on the combination of two algorithms. This method mostly uses the segmentation of tumor tissue with high accuracy and reproducibility comparable to manual segmentation. 


\subsection{PNN Techniques}

In this paper, they modified image segmentation techniques were applied on MRI scan images in order to detect brain tumors. in this paper, they modified Probabilistic Neural Network (PNN) model that is based on learning vector quantization (LVQ) with image and data analysis and manipulation techniques is proposed to carry out an automated brain tumor classification using MRI-scans[12]. The simulation results showed that the modified PNN gives rapid and accurate classification compared with the image processing and published conventional PNN techniques.

\subsection{Computer Aided System}

In this paper they propose a method for automatic brain tumor diagnostic system from MRI images. The system consists of three steps to detect and segment a brain tumor[13]. In the first stage, MR image of brain is acquired and pre-processing is done to remove the noise and to sharp the image. In the second step, global threshold segmentation is done on the sharpened image to segment the brain tumor. In the third step, the segmented image is post processed by morphological operations and tumor masking in order to remove the false segmented pixels. Results show that our propose technique accurately identifies and segments the brain tumor in MR images.

\section{PROPOSED SYSTEM}

$\mathrm{K}$-means algorithm is used to implement the segmentation of the MRI brain image. The Clustering is most important unsupervised learning problem as every other problem of this type of deals with finding a structure in a collection of data which is not labeled .A definition of clustering is the process of organizing objects into groups whose members are similar. The k-means algorithm is a process of iteration technique and it is used for partition the MRI into K cluster.

\section{CONCLUSION}

Many image processing technique have been initiated in the last decade. These techniques carry Content-Based Retrieval Technique, Component Labelling Algorithm, Fuzzy C-Mean Algorithm. However using these uncovered image processing data in the field of image segmentation is hard to implement and not so much effective. This is because some useful segmentation with high specificity, minimum accuracy. We argue that not all processing technique are useful. Hence misapprehension of image segmentation obtain from image processing lead to unsuccessful presentation. In this research work on effectual segmentation technique has been established to overcome the low rate of occurrence. The proposed system uses the two processed brain tumor detection and brain tumor segmentation in image processing.

\section{REFERENCES}

[1] Meiyan Huang,Wei Yang, YaoWu, Jun Jiang,Wufan Chen, Senior Member, IEEE, and Qianjin Feng*, Member, IEEE, IEEE TRANSACTIONS ON
BIOMEDICAL ENGINEERING, VOL. 61, NO. 10, OCTOBER 2014

[2] R. Manikandan, G.S. Monolisa and K. Saranya, A Cluster Based Segmentation of Magnetic Resonance Images for Brain Tumor Detection, Middle-East Journal of Scientific Research 14 (5): 669-672, 2013

[3] Atiq Islam, Syed M. S. Reza, and Khan M. Iftekharuddin*, Senior Member, IEEE, IEEE TRANSACTIONS ON BIOMEDICAL ENGINEERING, VOL. 60, NO. 11, NOVEMBER 2013

[4] Sahar Ghanavati1, Junning Li1, Ting Liu1, Paul S. Babyn2, Wendy Doda2,GeorgeLampropoulos11 AUG Signals Ltd., Toronto, ON., Canada2 Hospital for Sick Children, Department of Medical Imaging, University of Toronto, ON., Canada,2012

[5] Matthew C. Clark, Lawrence O. Hall,* Member, IEEE, Dmitry B. Goldgof, Senior Member, IEEE,Robert Velthuizen, Associate Member, IEEE, F. Reed Murtagh, and Martin S.Silbiger, IEEE TRANSACTIONS ON MEDICAL IMAGING, VOL. 17, NO. 2, APRIL 1998

[6] J.selvakumar, A.Lakshmi, T.Arivoli Brain Tumor Segmentation and Its Area Calculation in Brain MR Imagesusing K-Mean Clustering and Fuzzy C-Mean Algorithm.

[7] Ming-Ni Wu1, Chia-Chen Lin2, Chin-Chen Chang13 Brain Tumor Detection Using Color-Based K-Means Clustering Segmentation.

[8] Abduljawad A. Amory, Rachid Sammouda A Content Based Retrieval Method For MR Brain Images.

[9] Sahar Ghanavati1, Junning Li1, Ting Liu1, Paul S. Babyn2, Wendy Doda2,GeorgeLampropoulos1. Automatic Brain Tumor Detection in Magnetic Resonance Images.

[10] Matthew C. Clark, Lawrence O. Hall,* Member, IEEE, Dmitry B. Goldgof, Senior Member, IEEE,Robert Velthuizen, Associate Member, IEEE, F. Reed Murtagh, and Martin S. Silbiger."Automatic Tumor Segmentation Using Knowledge-Based Techniques".

[11] Hossam M. Moftah, Aboul Ella Hassanien, and Mohamoud Shoman." 3D Brain Tumor SegmentationScheme using K-mean Clustering and Connected Component Labeling Algorithms".

[12] Dina Aboul Dahab1, Samy S. A. Ghoniemy2, Gamal M. Selim3 Automated Brain Tumor Detection and Identification Using Image Processing and Probabilistic Neural Network Techniques.

[13] Akram, M.U. Dept. of Comput. \& Software Eng., Bahria Univ., Islamabad, Pakistan Usman, A. Computer aided system for brain tumor detection and segmentation. 